\title{
APPROACH MULTI-AGENTS EMBEDDED ALARM IN POTROOMS
}

\author{
Luis Carlos Costa Fonseca ${ }^{1}$, Alan Robert da Silva Ribeiro ${ }^{2}$ and Jonh \\ Selmo de Souza do Nascimento ${ }^{3}$ \\ ${ }^{1,2,3}$ Department of Computer Engineering and System, \\ State University of Maranhão, São Luis, Maranhão, Brazil \\ ${ }^{1}$ luiscarlos. uema@gmail.com \\ 2alan.robert2617@gmail.com \\ ${ }^{3}$ jonhselmo@pecs. uema.br
}

\begin{abstract}
Industrial Shop Floor environments require fast intervention of the controller's computers and operators to ensure high industrial production efficiency. This work focuses the electrolytic potrooms process control efficiency. The main goal of this work is to design an embedded solution for detection and alarm, using multi-agents system technologies so that controllers can alert plant operators about the problems in the electrolytic pot still malfunctioning after controller intervention. If the controller action was unsuccessful due to a feeder and pot bus problem, an audio alarm is immediately issued to the potrooms so that the operator can be notified about the specific problem, independently of the potrooms location.
\end{abstract}

\section{KEYWORDS}

Alarm, Potrooms, Agents, Embedded Systems, Pot

\section{INTRODUCTION}

In the aluminum production process, the cost and the quality of the product is strongly affected by the amount of alumina added to the pot production. The resistance and variations of first and second orders are used to assess the percentage of the alumina concentration in the bath [1]. The resistance variation is the primary variable used as an evaluation metric of vessel behavior. In practical terms, the monitoring and control of alumina in the bath changes are implemented in accordance with the assessment of the resistive variation. There are situations, however, where the electrolytic process control computers, even sending signals to the alumina feeding actuators and movement of buses that regulate the resistance of the electrolytic cells allow better control of the amount of alumina in the pot; this guarantee control may be interrupted by any mechanical failures in actuators and busbars.

Therefore, rapid actuation of the vessel operator to reestablishment of normal operation of the equipment and continuity of the aluminum production process with higher production gain becomes a daily preoccupation of management, to the extent that an aluminum production room comprises hundreds of devices distributed over large production halls. Figure 1 shows the Alumar

David C. Wyld et al. (Eds) : ICAITA, CDKP, CMC, SOFT, SAI - 2016

pp. 83- 95, 2016. (C) CS \& IT-CSCP 2016

DOI : 10.5121/csit.2016.61308 
potroom which has three production lines with 204, 250 and 256 electrolytic cells, each, for a total of 710 electrolytic cells.

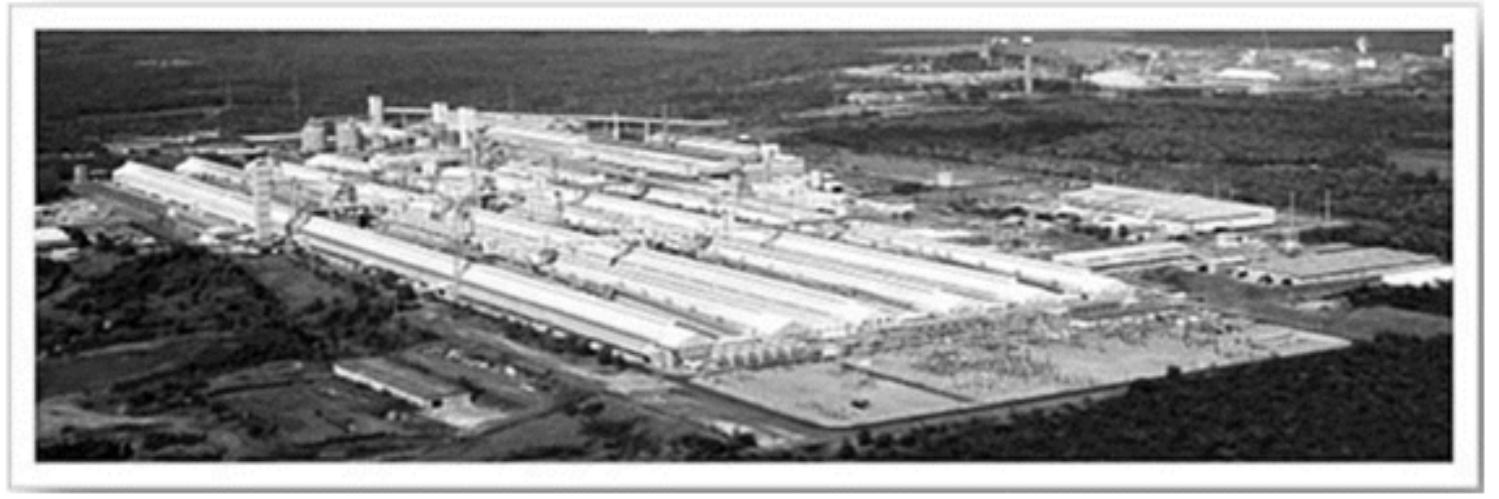

Figure 1. Reduction Alumar

Note that potrooms are arranged in large halls in which the electrolytic cells are disposed in series, side by side. These pots are controlled by the process control computers, which send signals to drive the feeders allowing it the addition of alumina in the pot. In addition, the control computer is responsible for sending signals to drive the mechanical drive bus, responsible for the regulation of the electrolytic tank resistance variation.

When, perhaps a mechanical failure occurs in one of these devices, we have as a consequence a delay in the correction and re-establishment of the operation of the electrolytic pot, causing the excess in the bus drive and alumina supply in the pot, due to unauthorized drives by computer control generating the accumulation of alumina or the lack of it, causing increased resistance to passage of electric current and negatively affecting the efficiency in aluminum production.

Thus, it becomes necessary to adopt a technique that best fits the application of electrolytic process control computer, in order to provide the necessary information and alerts and can thus overcome and minimize problems that may arise, for example, anode effect, unscheduled shutdown pot, bored pot, or quality time requirements and life for the potrooms.

Thus, an embedded solution with communications technology based on multi-agent systems is built based on stimulus-response model, in order to map and detect all abnormalities prevent the correct operation of the electrolytic pot, even with sending signals performance controllers. If signs of activity are not sufficient to establish the acceptable level of resistance change, and consequently, of alumina in the electrolytic pot, then audio alarms are generated immediately for operators and technicians of the potrooms (factory floor) can understand the situation and carry out the necessary intervention to solve the physical problem of equipment. The system is accessible and can be easily implemented in the automation industries that have controllers such as PLC (programming logic controller) and process control computer.

The technologies investigated in this research are the Process Control System, Multi-Agent Systems and Embedded Software, all used in the construction of a monitoring and real-time alarm solution. To perform this analysis, it is used as a case study alarm management system for aluminum production halls, rooms of tanks, which will be built using these technologies. 
Is approached, therefore, the need for detection and alarm automation environments and control in which controllers can not function correctly and precisely, due to mechanical problems in the equipment that receive the command signals for actuation, as the emitted pulses the controllers. In the case of large factory floor environments where each electrolytic tank requires their respective actuators work in real time, the extent to which the actuators are triggered, the delay in identifying problems of this nature can lead to loss of productivity and own electrolytic pot of aluminum production, generating as a result of damage to the production process.

The contribution of this article is to develop an embedded management solution and alarm based on the standard observe and react, using multi-agent systems for monitoring and conversion of messages generated by the controllers, allowing the broadcast in real time, state and environmental behavior, allowing adoption of preventive actions and operational in the shortest time interval, by the vats operators working there. It consists in building a software solution embedded in a raspberry PI plate for automation and control environment, based on the response stimulus model, able to communicate with controllers electrolytic cells through the use of multiagent systems, allowing communication between centralizing agents and controllers for transmitting / receiving text messages sent, generated by computer control, warning of each of the potential problems identified in several electrolytic cells. These text messages are received by the centralizing agent that converts audio messages transmitted to speakers of channels distributed over the potrooms, allowing the technician and in charge of the process in it can identify a pot with problem despite being in a distant location of the problem.

\section{CONTROL SYSTEM POTROOM}

In general, the process of aluminum production is based on the amount of alumina (A12O3), the raw material added to the electrolytic bath. In a bowl, electricity circulates between a positive (cathode) and a negative pole (anode). That of the alumina mixing process, existing electrolytic bath in the pot, and passing electric current anode cathode, it is apparent that liquid aluminum is removed from the vessel for the production of ingots. Figure 2 below shows the generic functional architecture of the aluminum production process, from the perspective of control systems. This architecture is applied to the modeling of a system of pots, specifically for monitoring purposes of their states and implementation of its controls.

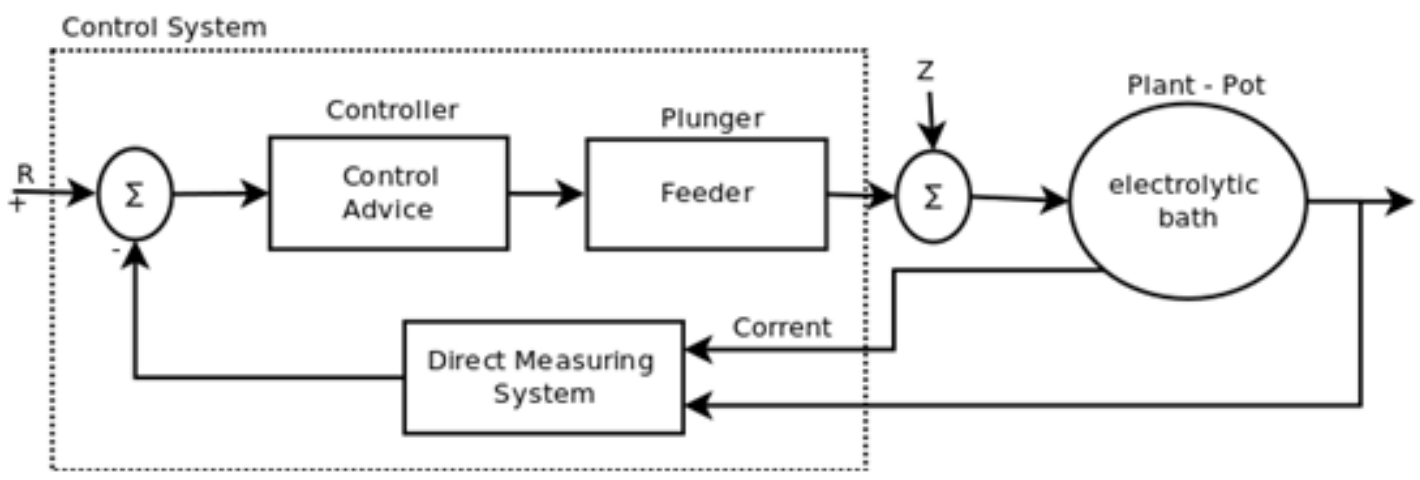

Figure 2. Block diagram for distribution of alumina in vessel 
The control system comprises sensor, controller and actuator. First, it is an indirect measurement system, because from voltage and current signals and other information such as operation status pot, to estimate quantities or rates those are used by the control device. The control system processes the signals and these are used to making decisions that interfere with the production process behavior. For example, control can promote actions to modify the frequency of operation of $\mathrm{A} 12 \mathrm{O} 3$ feeder.

This control system is run on computer called process control computer, and has as main objective to control the supply of alumina to the electrolytic cells, whether pre-baked type or Soderberg point-feeder [2]. The intention is to keep a quantity of alumina dissolved in the bath to ensure maximum production of aluminum. In pots of pre-baked type, the optimum concentration of alumina in the bath is around 3\% in the electrolytic bath. However, there are large variations in this concentration, depending on the operations carried out in vats and variations of other control parameters [3]. In practice, there is no method to directly measure this quantity or alumina concentration in the electrolytic bath in the process of speed control requires [4]. Therefore, the process computer controls the amount of alumina should meet the demand of alumina is added to the vessel within the rated power range. The feed interval set in seconds, is the time window in which the computer will send a pulse to drive the alumina feeders, wherein said range is from pot to pot. It means that each production cell (electrolytic tank) has its power gap target.

When the control computer sends a signal to drive the feeders, and for some reason, such feeders are not triggered, it means that some physical problem may be occurring. In this case, a physical intervention such equipment is required. For this task, they are designated process technicians who perform the repair and the reestablishment of the operation of the feeders and voltage busbars. The problem becomes complex when technicians deal in a potroom environment with large amounts of electrolytic cells arranged in large rooms and corridors in an aluminum production plant.

\section{MULTI-AgENT SYSTEMS}

The definition of an agent can be expressed in many different ways. [5] defines an agent as a computational entity software, located in a given environment, which has the perception that environment via sensors has reasoning ability and acts autonomously in that environment through actuators so to perform a specific function for which it was designed. [6], defined as something that can be seen as having the perception of an environment through sensors and acting in this environment through actuators. For [7] would be a piece of hardware or a computer system based on software that enjoys some properties, such as learning autonomy, responsiveness, pro-activity and social skills.

Figure 3 shows the interaction between two or more agents in a computer system and configures a Multi-Agent System where these work together to perform certain tasks or perform a set of goals. Scientific research and the practical implementation of Multi-Agent Systems is focused on building standards, principles and models that allow the creation of small and large companies of semi-autonomous agents, able to conveniently interact in order to achieve their objectives [8]. 


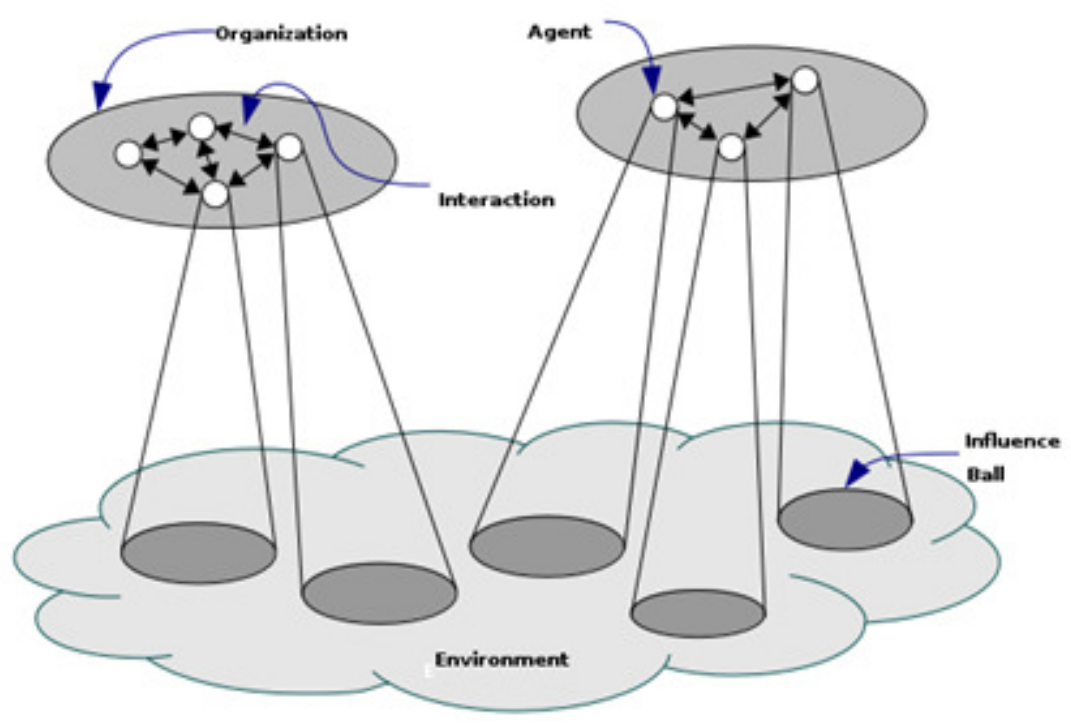

Figure 3. Structure of a Multi-Agent System

\subsection{Jade}

JADE tool (Java Agent Development Framework) began in 1998 through a CSELT (Telecom Italia), whose development was motivated by the need for validation of specifications of FIPA (Foundation for Intelligent, Physical Agents). AvJADE in 2000, became an open source platform, being distributed by Telecom Italia on the LGPL (Library Gnu Public License) [9].

JADE provides certain features, such as distributed applications that exploit the use of software agents. Being built on the Java platform, enables deployed agents using the JADE framework, which can run on any operating system (OS), which makes it ideal for environments where you can not get information about the OS used [10].

Other JADE's features are the development of agents and control of them via a graphical interface [9].

\subsection{Auml}

Unified Modeling Language Agent (Auml) is a standardized graphical modeling language by FIPA (Foundation Intelligent Physical Agents). Auml was proposed as an extension of UML (Unified Modeling Language), which uses decomposition, abstraction and organization to reduce the complexity of software development, decomposing a system in small parts of objects, models, use case or class, several operational actions. In relation to abstraction, it provides a specialized abstract view of modeling (class, use case diagram of interface etc.) and is used to create a set of semantics and operating service conditions and infrastructure [11].

The Auml offers structures as a class diagram and interface to show how agents can be erected in an agent system. The model focuses on a point at a time, increasing the ability to understand the issues to complex problems during the lifetime of the system design. 
The main parts of Auml are the mechanisms for modeling multi-agent interaction protocols. This is accomplished by introducing a new class diagrams UML diagrams protocol. These extend diagrams and sequence diagrams of UML state, as shown in Figures 4 and 5.
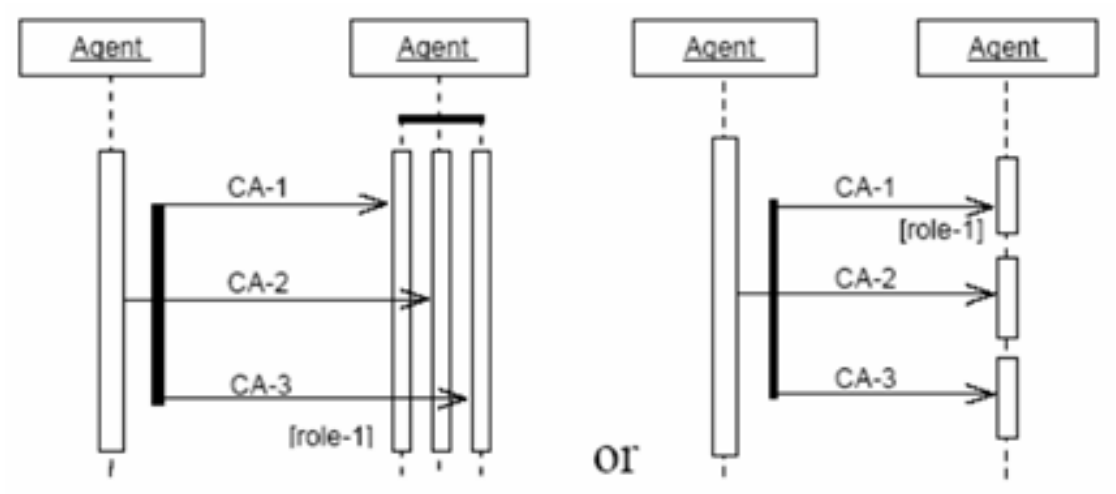

Figure 4. AUML - Diagram Sequence

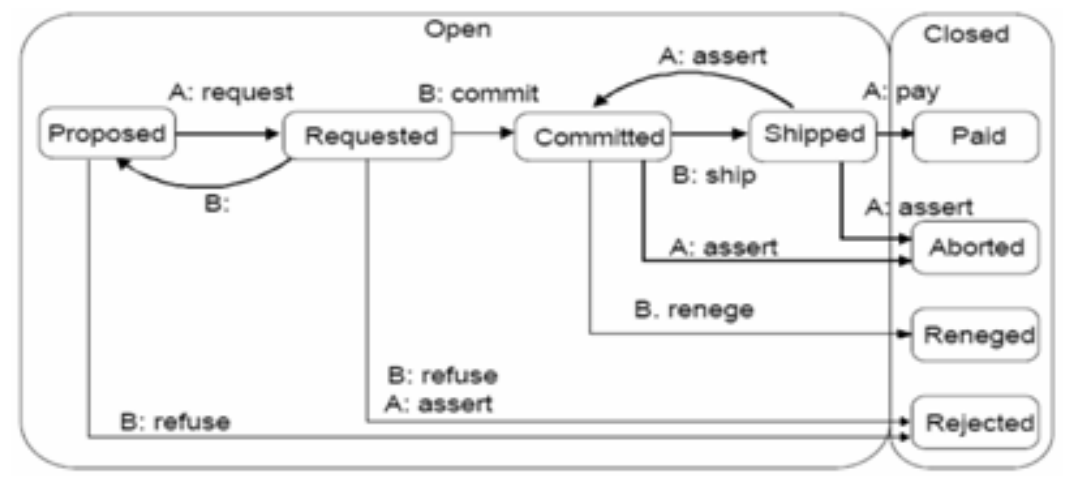

Figure 5. AUML - State Diagram

\section{EMBEDDED SYSTEMS}

The embedded systems are a combination of computer hardware and software, sometimes having mechanical parts designed to perform a dedicated function, wherein such systems are often only a small part of a larger system [12].

Typically, they are implemented from different technologies such as microprocessors, microcontrollers, DSP, reconfigurable circuits, analog circuits and microwave and even microelectromechanical systems (MEMS - Micro Electro Mechanical Systems) [13].

\subsection{Raspberry PI}

The Raspberry Pi model B is a small computer that uses the Broadcom BCM2835 multimedia processor, the SoC type (system-on-chip), with $700 \mathrm{MHz}$ 32-bit, built on the ARM11 architecture [10]. The board is fed by a micro-USB port, with $5 \mathrm{~V}$ voltage and requiring at least $700 \mathrm{~mA}$, with waste of energy varying in $3.5 \mathrm{~W}$ [14]. 
The Raspberry Pi, as shown inFigure 6, has no hard disk on your hardware composition, using only one memory card. For operating system installation it is necessary that the card has at least 2 GB capacity for all the necessary files. The operating system commonly used is the Raspibian, a distribution recommended by Raspberry Foundation, based on Debian [15].

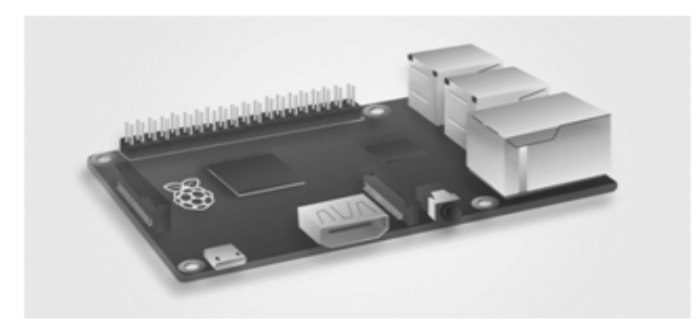

Figure 6. Raspberry B+ model

\section{RELATED WORK}

Binnicker [16] in 2015 presents a tool to detect and alert with temperature sensors for vehicles occupied by passengers. The system alerts drivers if a child is left unattended in the vehicle in order to prevent the child from being exposed to high or low temperatures inside the vehicle. To this end, sensors are used to capture data via occupancy sensors, temperature and distance. The data captured by the sensors determine the need to alert sent to the vehicle owner (driver) if an occupant has been left inside the vehicle, still controlling some functions of the vehicle, allowing foreign individuals to rescue him, with the unlocking of doors, windows opening, with possible activation temperature of the automobile system.

M. Kumar et al. in [17] designed and developed an intelligent detection and intrusion alert system designed to increase security, with real positive identification probability of attackers and intruders, compared to other electronic security systems already used. Through multiple sensors, the system evaluates the extent of danger exhibited by a person or animal within the confines of the residence, conveying the owner of the residence, the various information about critical events generated.

Ramya [18] presents a construction work involving containing a microcontroller system for detecting and warning of toxic gases, particularly propane and LGP. Specific sensors for identification of these two noxious gases are added to the microcontroller, which through the use of analog / digital converter capture and analyze the information, which are also shown in the display. If the gases exceed acceptable limits, the system immediately generates an alarm and also send SMS alert message to authorized persons using GSM technology.

Gaspar et. al. [19] addresses the adoption of an alarm management philosophy for incident management that avoids the generation of a huge amount of alarms in order to make life easier for technicians who must manage in real time complex and dynamic environments and good management alarms can provide crucial information to identify the cause of the fault and replace the plant in normal operating state.

Morales [20] created and patented a monitoring and alarm system in a network audio link that detects sound alarms triggered and inform the responsible authorities. The system and method 
created based on the audio produced from alarms which are triggered. Implemented for PC type computers or other premises of processors, different sound coming from different alarms, classifies the sound as a specific alarm by FFT processing and distributes the alarm over the Internet, public telephone switches, or other communication links to central station, which then distributes the alarm to the authorities. In another embodiment, the alarm reports are generated directly from the PC to the residence or specific company where authority is located.

\section{PRELIMinary ReSUlts}

As preliminary results, diagrams were built in Agent UML (Auml) for multi-agent embedded proposed solution, which includes the deployment diagram, use cases, activities, block, and an embedded prototype raspberry pi with jade platform running on environment simulation, where the centralizing agent is embedded in the board and the controlling agents are available in environments that simulate controllers. All diagrams were constructed using the free tool-day standard 0.97.2 (http://live.gnome.org/Dia). The source code of the solution multi-agent was developed in Java with Netbeans IDE version 8.1, where the environment is multi-agent on the implementation platform JADE, in version 4.4.0.

\subsection{Diagram Use Cases}

Figure 7 shows the actors are placed in the context of the proposed approach and its use cases, showing the set of paths that agents can run through the system, each with a discrete goal. The actors representing the controlling system of tanks in practice can be represented by any controller technology, this PLC (programmable logic controllers) to real-time vats control applications that interact with Analog Stick / digital converters, analog and digital cards Mustangs.

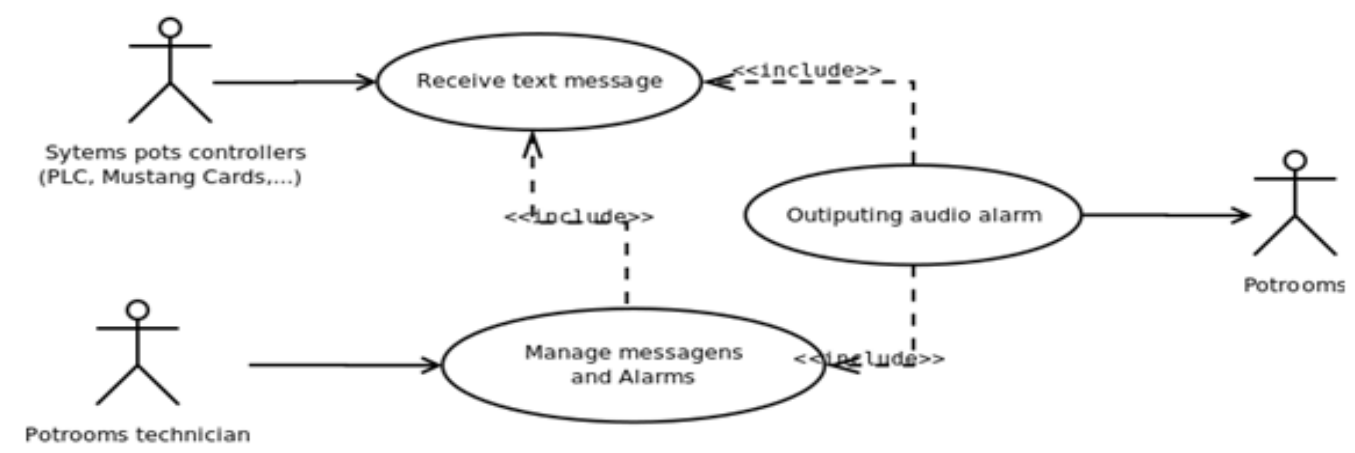

Figure 7. Diagram Use Cases of the proposed system

The use case Send audio alarm, converts the textual words received by dividing each of them into audio files (eg. .wav) that are sent to their rooms vats according to the parameter that identifies the number of pot with problem.

\subsection{Transition State Diagram}

The Transition State Diagram shown in Figure 8 represents the state and associated transitions to the communication protocol between the developed agents and between the controller and agents, 
which at the end of processing, transfer to the vat room audible alarm identifying a potential problem in an electrolytic tank. Is represented in this case, the level of interaction between players by exchanging messages and activation through behaviors.

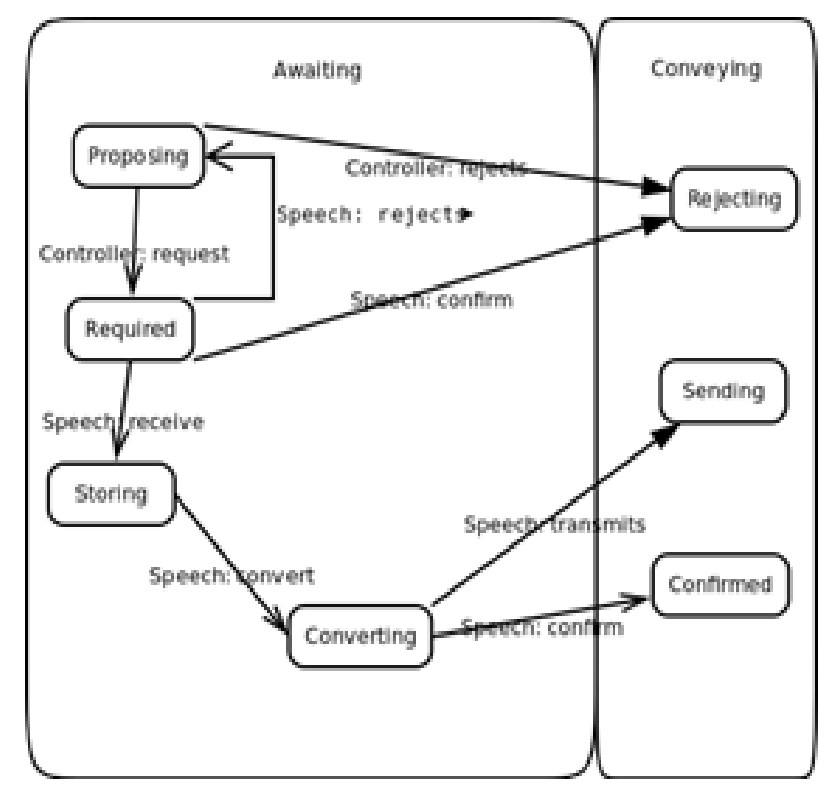

Figure 8. State Transition Diagram of the Proposed System

Through this diagram, we see the main actors interacting in the sending and receiving of text messages parameterized (Controller and Speech) that is validated, converted to audio and broadcast.

\subsection{Diagram Deployment}

The deployment diagram shown in Figure 9 detailing the distribution of the proposed solution in a generic environment potroom controlled by the controlling computer. It is observed that the bundled solution raspberry pi plate is arranged in the process control network, in the same environment controllers. The audio channel raspberry card is connected to speakers arranged in the potroom. Therefore, the power pulses, ascent and descent bus, when they jammed by mechanical failures in the vats can be signaled in the form of beep, in which the solution proposal report to the operator exactly what existing physical problem.

\subsection{Block Diagram of the Proposed Solution}

Each controller agent is responsible for receiving the parameterized messages generated by the control computer in which the agent is inserted. From the generation and reception of such text messages, with the use of multi-agent platform JADE, the controlling agents send text messages with the problem identified in a given tank to the centralizing agent, which queues the message and is in charge of is converted it audio, transferring them to the speakers of the respective room vats, where the pot with problem lies. The solution proposed is the block diagram shown in Figure 10. 


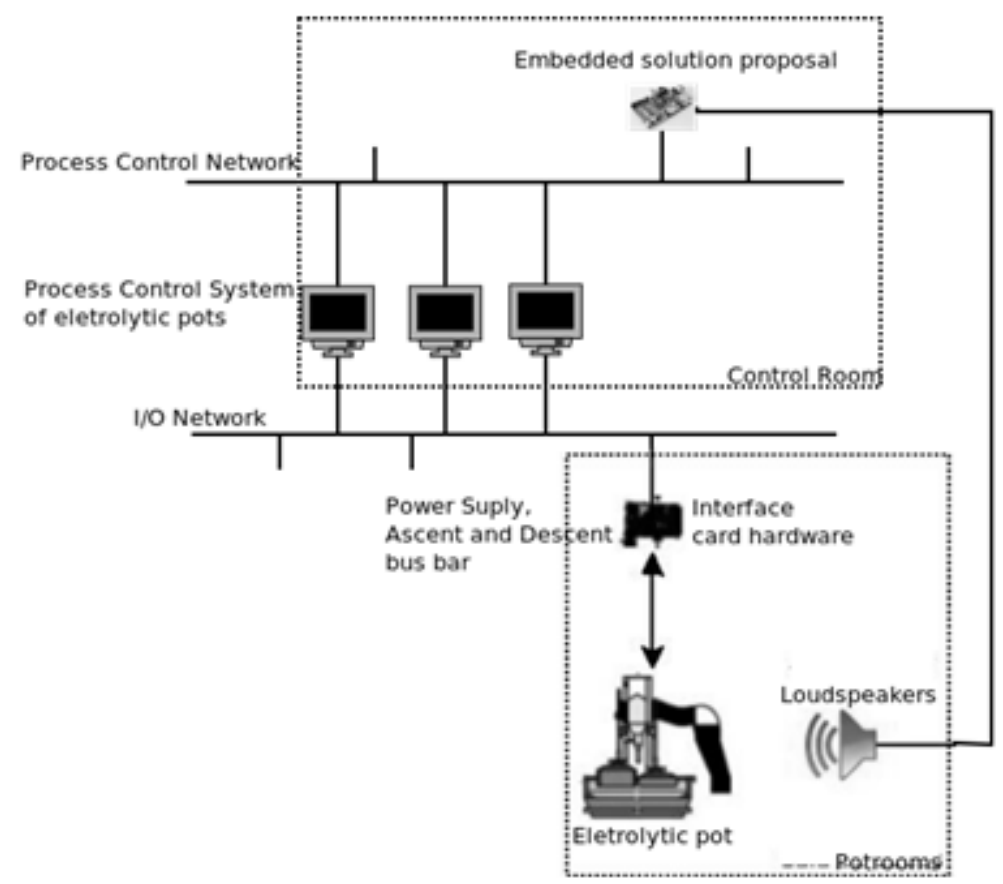

Figure 9. Deployment Diagram of the Proposed System

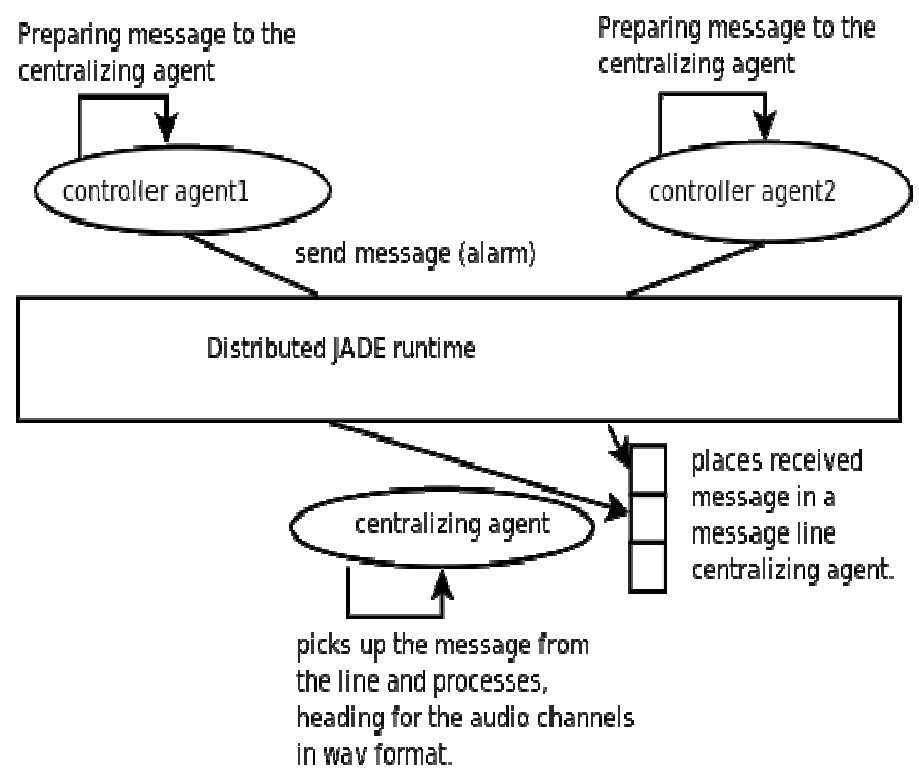

Figure 10. Solution Block Diagram proposal 


\section{IMPLEMENTATION}

Alarms tanks with high voltage, much increase and / or bus down, swings, and its operating status were detected by the controlling agents and sent to the centralizing agent of the respective potroom, residing in raspberry pi. The centralizing agent identifies the queue textual alarm messages that are sent in FIFO order (first-in, first-out) to the potroom in an audible format, as a warning system, which helps in faster diffusion of the critical situation found. The prototype of the proposed system is shown in Figure 11.

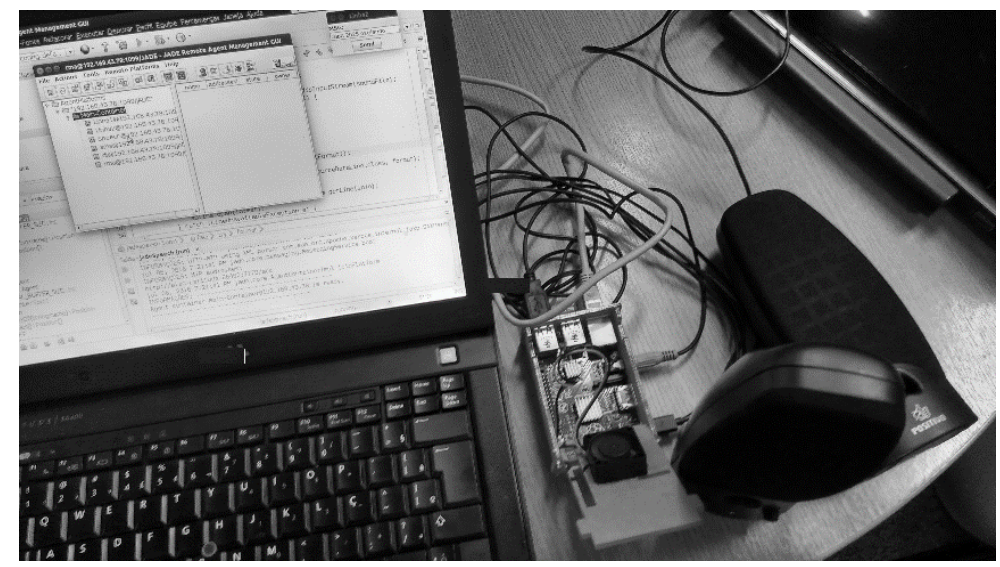

Figure 11. Prototype of the Proposed System

\section{CONCLUSiOnS}

An embedded system using multi-agent platform for detection and alarm electrolytic cells in potrooms was implemented. For demonstration purposes they were made simulation controllers pot and controlling and centralizing agents responsible the generation and transmission of alarms in audio format for the tanks room, respectively. The extent to which the controllers identify some kind of problem in a particular pot, textual message of the problem in question, parameterized for the pot number identifier is generated and transmitted to the controller agent forwards to the centralizing agent. This solution may be suitable for any shop floor environment where there is a process automation for large equipment and controllable mechanisms that need to maximize their production with minimal stop as a function of real problems identified the equipment, enabling operators and technicians act to minimize downtime.

\section{REFERENCES}

[1] Fiona, Stevens McFaden \& Bearne, Geoffrey (2001) Apliccation of advanced process control to aluminium reduction cell - a review, TMS- The Mineral, Metal \& Materials Society.

[2] Braga, Carlos A. P., Martin, S., Nagem N. F, Ritter, C., Silva, Ari, Verlihay, Mark (2007) Faster Anode Effect Kill. TMS Letters, v. 50, p. 417-422.

[3] Silva, Ari (1995) Alumina feed control system of line 1 and line 2 potrooms at ALUMAR. ABAL Associação Brasileira de Alumínio. 
[4] Silva, Ari, Carlos Braga, Eliezer Batista (2002) Smelter personnel technical training on aluminum production. ALUMAR - Consórcio de Alumínio do Maranhão.

[5] Reis, Luis Paulo (2003) Coordination in Multi-Agent Systems: Applications in University Management and Robotic Soccer. PhD, Electrical and Computers Engineering, FEUP, Porto.

[6] Russel, Stuart. "NORVIG, Peter." (2013) Inteligência Artificial. Editora Campus. Tradução da 3a edição.

[7] Wooldridge, Michael, and Nicholas R. Jennings (1995) Intelligent agents: Theory and practice. The knowledge engineering review 10.02: 115-152.

[8] Lesser, Victor (1999) Cooperative Multi-Agent Systems: A Personal View of the State of the Art, IEEE Transactions on Knowledge and Data Engineering, Vol. 11, $\mathrm{N}^{\circ} 1$.

[9] Telecom Italia - JADE - http://jade.tilab.com/

[10] Bellifemine, F., G. Caire, D. Greenwood, e I. NetLibrary (2007) Developing Multi-agent Systems with JADE. Springer

[11] Gatti, M., Von Staa, A., Lucena, C. (2007) AUML-BP: A Basic Agent Oriented Software Development Process Model Using AUML., PUC-Rio, n. 21/07, p. 25.

[12] William, Stallings (2010) Arquitetura e Organização de Computadores.

[13] Dias, Ailton Fernando (2001) "Concepção Conjunta Hardware/Software de Sistemas Embarcados de Processamento de Imagens." CDTN/CNEN. Belo Horizonte, Brasil.

[14] Richardson, Matt, and Shawn Wallace (2013) "Primeiros passos com o Raspberry Pi." Primeira Edição. Novatec Editora Ltda.

[15] Raspberry PI Foundation: http://www.raspberrypi.org/downloads/

[16] Binnicker, G. (2015) Temperature-Sensitive Vehicle Occupancy Detection and Alert System. https://www.google.com/patents/US9428109

[17] S. Kumar, et al. (2015) "A Pattern Matching Model For Misuse Intrusion Detection,", pp. 1-11, (found at http://www.cs.purdue.edu/coast/archive/data/categ24.html).

[18] Ramya V., Palaniappan B. (2012) Embedded system for Hazardous Gas detection and Alerting. International Journal of Distributed and Parallel Systems (IJDPS) Vol.3, No.3.

[19] Gaspar, D., Ferreira, D., Coutinho, J., Correia, J., Albuquerque, P. (2010). A Política de Alarmes na Gestão da Manutenção de uma Indústria Farmacêutica. Millenium, 39: 135-151.

[20] Morales, F. (2001) "Network audio-link fire alarm monitoring system and method" https://www.google.com/patents/US6215404

[21] Braga, Carlos A., Nagem,N.F, Fonseca, J. V. (2007) "Bandwidth adjustment of digital kalman filter for indirect measurement of electrolytic bath state variables". 2nd International Conference on Sensing Technology. 


\section{AUTHORS}

Luis Carlos Fonseca graduated in Technology in the Data Processing of Maranhão University (2000), Master in Engineering Electricity from Federal University of Maranhão (2003) and PhD in Computer Science in Education from the Federal University of Rio Grande do Sul (2009). He is currently assistant professor at the State University of Maranhão. He has experience in computer science with an emphasis in Information Systems, mainly in the following areas: Artificial Intelligence, Information Technology in Education, Software Engineering, Information Retrieval, Recommender Systems.

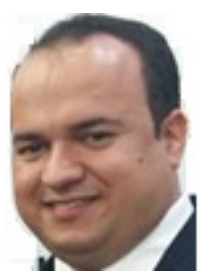

Alan Robert da Silva Ribeiro graduated in Bachelor of Computer Science from Federal University of Maranhão (1999), specialization in Systems Analysis and Design from the Federal University of Maranhão (2006), specialized in Advanced Systems for Internet and Intranet at University Center Maranhão (2007) and is Masters Student of Computer Engineering and Systems at the State University of Maranhão. It is currently a Ministerial Analyst and Coordinator of the Public Ministry of the Information Technology area of the state of Maranhão, having worked as a Senior Process Control Systems Analyst at multinational ALCOA, which was part of SDG global group (Service Delivery Group), responsible for development and installation of process control systems in the company's factories in the world. He has experience in the area of Computer Science, Aluminum Process Control, Information Technology Management, acting on the following topics: Electrolytic Control Computer, Artificial Intelligence, Information Technology Governance, Database, Ubiquitous Computing and Driven Engineering by models.

John Selmo de Souza do Nascimento holds a degree in Computer Engineering with emphasis on Automation and Process Control at the State University of Maranhão (2015). Currently is Masters Student of Computer and Systems Engineering from State University of Maranhão. He has experience in Geosciences with an emphasis on Georeferenced systems, robotics, mechatronics and automation.

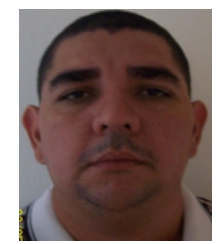

